Article available at http://www.parasite-journal.org or http://dx.doi.org/10.1051/parasite/1997041049

\title{
IMPORTANCE DU PARASITISME DANS LA COMMUNAUTÉ de Gastéropodes de l'étang de Combourg (Bretagne, france)
}

\author{
GÉRARD C.*
}

Summary : IMPORTANCE OF PARASITISM IN THE COMMUNITY OF GASTROPODS IN THE POND OF COMBOURG (BRITAIN, FrancE)

The community of Gastropods in the pond of Combourg is composed of 15 species among Pulmonates (Planorbidae, Lymnaeidae, Physidae, Ancylidae) and Prosobranches (Valvatidae), and the community of Trematodes of nine species (described through cercariae). The rate of parasitism is null concerning seven species of snails and varies from 1 to $80 \%$ concerning the eight others. We have demonstrated with the help of a significative model that the frequency of Gastropods is all the more little than the prevalence is high, then a possible regulation of the molluscan host populations by larval Trematodes.

KEY WORDS : community, Gastropods, Trematodes, prevalence, regulation.

\section{INTRODUCTION}

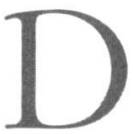
epuis une dizaine d'années, on constate un intérêt croissant de certains écologues pour le rôle des parasites dans les processus de régulation des populations hôtes (Anderson, 1978, 1979; Anderson \& May, 1978; Holmes, 1982; May, 1983; Keymer et al., 1991; Combes, 1993). Si un grand nombre d'investigations expérimentales suggèrent que les parasites peuvent en pratique être des régulateurs (Scott \& Anderson, 1984; Lauckner, 1986; Scott, 1987; Keymer et al., 1991; Lafferty, 1993a), seuls quelques travaux ont démontré leur impact sur les populations naturelles, dont le plus célèbre et le plus convaincant concerne le modèle Trichostrongylus tenuis/Lagopus lagopus scoticus (Hudson et al., 1985; Dobson \& Hudson, 1986, 1992; Hudson \& Dobson, 1992).

Les connaissances expérimentales sur les associations Trématodes/Mollusques, en particulier sur la pathologie des Mollusques parasités (traits de vie) (pour revues : Thompson, 1985; Bayne \& Loker, 1987; Hurd, 1990), permettent d'envisager en théorie un rôle régulateur des Trématodes larvaires vis-à-vis des populations de Mollusques hôtes. Nous avons réalisé une étude pré-

\footnotetext{
* Laboratoire de Zoologie et d'Écophysiologie, UA INRA/UMR CNRS n 1853, Avenue du Général Leclerc, 35042 Rennes cédex, France. Correspondance : Claudia Gérard.

Tel. : (33) 0299281444 - Fax : (33) 0299281612.
}

Résumé :

La communauté de Gastéropodes de l'étang de Combourg comprend 15 espèces parmi les Pulmonés (Planorbidae, Lymnaeidae, Physidae, Ancylidae) et les Prosobranches (Valvatidae); la communauté de Trématodes en comprend neuf, décrites à partir des cercaires. Le taux de parasitisme par les Trématodes est nul pour sept espèces de Mollusques et varie de 1 à $80 \%$ pour les huit autres. Nous avons montré à l'aide d'un modèle significatif que la fréquence des Gastéropodes est d'autant plus faible que la prévalence est élevée, d'où une régulation possible des populations de Mollusques hôtes par les Trématodes larvaires.

MOTS CLÉS : communauté, Gastéropodes, Trématodes, prévalence, régulation. liminaire des peuplements de Gastéropodes d'un écosystème dulcicole en relation avec la présence de Trématodes larvaires (sporocystes ou rédies et cercaires). Cette première approche en milieu naturel a pour but de mettre en évidence les populations de Gastéropodes pour lesquelles le parasitisme est susceptible de constituer un facteur régulateur déterminant (ou tout au moins non négligeable).

\section{MATÉRIELS ET MÉTHODES}

T e site de cette étude est l'étang de Combourg en Ille-et-Vilaine (latitude Nord $48^{\circ} 24^{\prime}$, longitude Ouest $1^{\circ} 45^{\prime}$, superficie $\geq 20$ ha), étendue d'eau stagnante peu profonde. La zone échantillonnée est une bande littorale de $100 \mathrm{~m}$ de long, de $2,5 \mathrm{~m}$ de large et de profondeur maximum de $1,0 \mathrm{~m}$.

Deux prélèvements ont été réalisés le $1^{\text {er }}$ décembre 1994 et le 12 mars 1995 au cours desquels les Mollusques (1 365 individus au total) ont été collectés pendant 2 heures (10-12 h) à l'aide d'un filet-troubleau (maille de nylon de $1 \mathrm{~mm}$, ouverture carrée de $50 \mathrm{~cm}$ de côté) passé dans toute la colonne d'eau. Tous les Gastéropodes ont été ensuite identifiés au laboratoire, mesurés au pied à coulisse (précision $=0,1 \mathrm{~mm}$ ) : hauteur pour les coquilles coniques, diamètre pour les coquilles discoïdes, puis disséqués sous loupe binoculaire afin de vérifier la présence de parasites. Les sporocystes ou les rédies, et les cercaires en cas d'infec- 
tion patente, ont été examinés au microscope optique. Le test $\mathrm{U}$ de Mann-Withney $(p=0,05)$ a été utilisé pour comparer les tailles des Mollusques sains et parasités.

\section{RÉSULTATS}

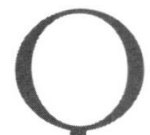

uinze espèces de Gastéropodes ont été trouvées dans l'étang de Combourg (tableau I), elles appartiennent à cinq familles différentes - une seule famille de Prosobranches, les Valvatidae, représentée à $95 \%$ par Valvata cristata, et quatre familles de Pulmonés Basommatophores : les Planorbidae, les Lymnaeidae, les Physidae et les Ancylidae par ordre décroissant d'importance. Sur l'ensemble du peuplement de Gastéropodes (1 365 individus échantillonnés), la famille des Planorbidae est la mieux représentée en nombre d'espèces (6) et en nombre d'individus (1 020); Gyraulus albus est l'espèce la plus abondante et constitue à elle seule environ $67 \%$ des Gastéropodes prélevés dans l'étang.

Sur les 15 espèces de Gastéropodes échantillonnées, sept n'ont jamais été trouvées parasitées : Lymnea auricularia, Lymnaea palustris, Gyraulus laevis, Armiger crista, Segmentina nitida, Physa acuta, Ancylus lacus-

\begin{tabular}{|c|c|c|c|c|c|c|}
\hline & \multicolumn{3}{|c|}{ Effectif } & \multicolumn{3}{|c|}{ Fréquence } \\
\hline & Déc. & Mars & Total & Déc. & Mars & Moyenne \\
\hline \multicolumn{7}{|l|}{ Prosobranches } \\
\hline Valvatidae & & & & & & \\
\hline Valvata cristata & 202 & 94 & 296 & 23,43 & 18,73 & 21,08 \\
\hline Valvata pulchella & 6 & 4 & 10 & 0,70 & 0,80 & 0,75 \\
\hline Valvata piscinalis & 3 & 2 & 5 & 0,35 & 0,40 & 0,37 \\
\hline \multicolumn{7}{|l|}{ Pulmones } \\
\hline Planorbidae & & & & & & \\
\hline Gyraulus albus & 557 & 356 & 913 & 64,62 & 70,92 & 67,77 \\
\hline Planorbis planorbis & 50 & 11 & 61 & 5,80 & 2,19 & 4,00 \\
\hline Planorbarius corneus & 8 & 2 & 10 & 0,93 & 0,40 & 0,66 \\
\hline Armiger crista & 3 & 27 & 30 & 0,35 & 5,38 & 2,86 \\
\hline Segmentina nitida & 4 & 0 & 4 & 0,46 & 0,00 & 0,23 \\
\hline Gyraulus laevis & 2 & 0 & 2 & 0,23 & 0,00 & 0,12 \\
\hline \multicolumn{7}{|l|}{ Lymnaeidae } \\
\hline Lymnaea peregra & 6 & 0 & 6 & 0,70 & 0,00 & 0,35 \\
\hline Lymnaea stagnalis & 5 & 0 & 5 & 0,58 & 0,00 & 0,29 \\
\hline Lymnaea palustris & 2 & 0 & 2 & 0,23 & 0,00 & 0,12 \\
\hline Lymnaea auricularia & 1 & 0 & 1 & 0,12 & 0,00 & 0,06 \\
\hline \multicolumn{7}{|l|}{ Ancylidae } \\
\hline Ancylus lacustris & 9 & 6 & 15 & 1,04 & 1,20 & 1,12 \\
\hline \multicolumn{7}{|l|}{ Physidae } \\
\hline Physa acuta & 5 & 0 & 5 & 0,58 & 0,00 & 0,29 \\
\hline Total & 863 & 502 & 1365 & & & \\
\hline
\end{tabular}

Tableau I. - Communauté de Gastéropodes de l'étang de Combourg en décembre 1994 et mars 1995. tris; nous considérons qu'elles n'appartiennent pas au spectre d'hôtes des Trématodes présents dans l'étang, ou bien que les prélèvements ont été effectués en dehors de la période de recrutement des Trématodes par les Mollusques.

Parmi les huit espèces de Mollusques trouvées parasitées (tableau II, fig. 1), on constate des taux de prévalence très différents (de 1 à $80 \%$ ) selon les espèces et la période de l'année. Nous avons montré l'existence d'un modèle significatif (coefficient de Spearman, $r=0,82, p=0,01)$ mettant en relation la prévalence et la fréquence des Mollusques : prévalence $=39,81$ $\times 10^{-0,023}$ fréquence. Plus une espèce hôte présente un taux de prévalence élevé et moins elle est fréquente dans le peuplement de Gastéropodes (fig. 2). D'autre part, la taille des Mollusques parasités (tableau III) n'est pas significativement différente de celle des Mollusques sains (test U).

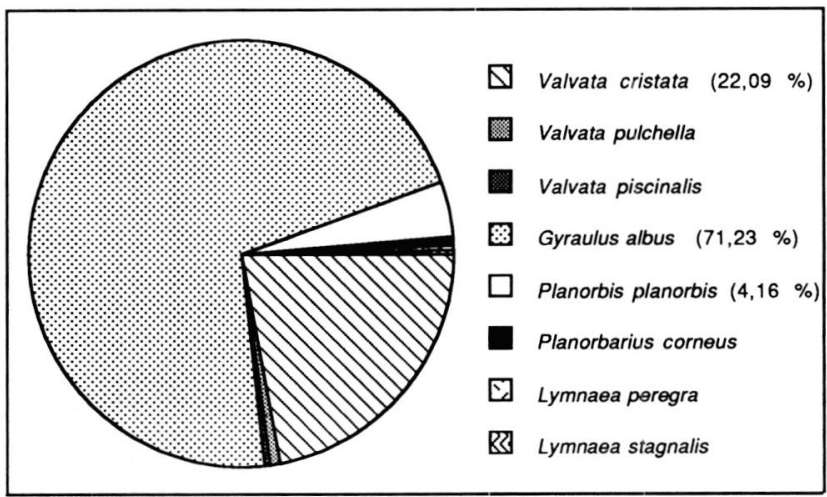

Fig. 1. - Communauté de Gastéropodes présentant des Trématodes : fréquence des espèces (\%).

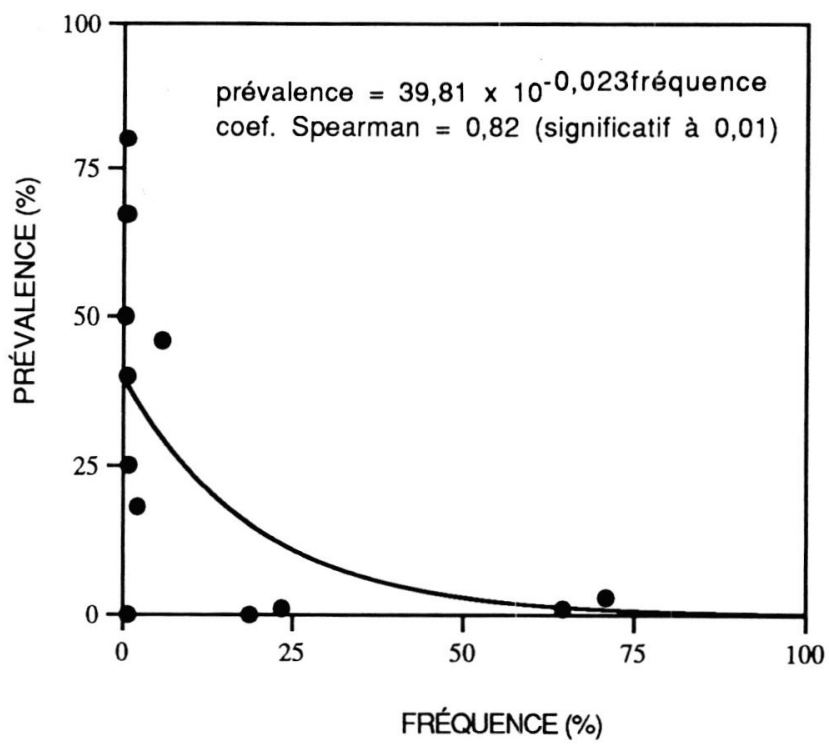

Fig. 2. - Relation entre la fréquence des espèces de Mollusques parasitées et leur prévalence. 


\begin{tabular}{|c|c|c|c|c|c|c|}
\hline & \multicolumn{2}{|c|}{ Effectif } & \multicolumn{3}{|c|}{ Prévalence \% } & \multirow{2}{*}{$\begin{array}{c}\text { Types de cercaires } \\
+ \text { (nombre d'individus hôtes) }\end{array}$} \\
\hline & Déc. & Mars & Déc. & Mars & Moyenne & \\
\hline \multicolumn{7}{|l|}{$\begin{array}{l}\text { Prosobranches } \\
\text { Valvatidae }\end{array}$} \\
\hline $\begin{array}{l}\text { Valvatidae } \\
\text { Valistata }\end{array}$ & 2.00 & 0,00 & 1.00 & 0.00 & 0.50 & $C(2)$ \\
\hline Valvata pulchella & 2,00 & 0,00 & 40,00 & 0,00 & 20,00 & $C(2)$ \\
\hline Valvata piscinalis & 2,00 & 1,00 & 67,00 & 50,00 & 58,50 & $C(3)$ \\
\hline \multicolumn{7}{|l|}{ Pulmones } \\
\hline Planorbidae & & & & & & \\
\hline Gyraulus albus & 5,00 & 11,00 & 1,00 & 3,00 & 2,00 & C (14), F1 (1), N (1 \\
\hline Planorbis planorbis & 23,00 & 2,00 & 46,00 & 18,00 & 32,00 & C (5), X1 (16), E (4) \\
\hline Planorbarius corneus & 2,00 & 1,00 & 25,00 & 50,00 & 37,50 & $\mathrm{X} 1(3)$ \\
\hline Lymanaeidae & & & & & & \\
\hline Lymnaea peregra & 4,00 & & 67,00 & & 67,00 & $\mathrm{X} 2$ (2), X3 (2), F2 (2), F3 (2) \\
\hline Lymnaea stagnalis & 4,00 & & 80,00 & & 80,00 & $\mathrm{E}(1), \mathrm{F} 3(3)$ \\
\hline
\end{tabular}

Cinquante-neuf individus parasités sur 1365 mollusques : 4,32\% de prévalence globale (C : cercariaea, E : échinostome, F : furcocercaire, $\mathrm{N}$ : notocotyle, $\mathrm{X}$ : xiphidiocercaire).

Tableau II. - Mollusques parasités de la communauté de Gastéropodes de l'étang de Combourg en décembre 1994 et mars 1995.

\begin{tabular}{|c|c|c|}
\hline & $\begin{array}{c}\text { Mollusques } \\
\text { sains }\end{array}$ & $\begin{array}{l}\text { Mollusques } \\
\text { parasités }\end{array}$ \\
\hline Valvata cristata & $2,95 \pm 0,28$ & $3,9 \pm 0,10$ \\
\hline Valvata pulchella & $2,45 \pm 0,04$ & $2,45 \pm 0,05$ \\
\hline Valvata piscinalis & $4,05 \pm 1,55$ & $2,50 \pm 0,57$ \\
\hline Gyraulus albus & $4,52 \pm 0,15$ & $3,64 \pm 0,34$ \\
\hline Planorbis planorbis & $11,48 \pm 0,37$ & $11,65 \pm 0,29$ \\
\hline Planorbarius corneus & $11,83 \pm 1,38$ & $23,17 \pm 3,84$ \\
\hline Lymnaea peregra & $13,87 \pm 3,76$ & $16,7 \pm 1,47$ \\
\hline Lymnaea stagnalis & $47,5^{*}$ & $40,38 \pm 4,42$ \\
\hline
\end{tabular}

${ }^{*}$ Un seul individu sain pour L. stagnalis.

Tableau III. - Comparaison des tailles moyennes des Mollusques sains et parasités (en mm, moyenne \pm écart-standard) pour les neuf espèces présentant des Trématodes.

En ce qui concerne la communauté de Trématodes de l'étang de Combourg, neuf types de cercaires ont été observés (tableau IV) : trois xiphidiocercaires (X1, X2, $\mathrm{X} 3$ ), trois furcocercaires (F1, F2, F3), un échinostome (E), une cercaire notocotyle $(\mathrm{N})$ et une cercaire sans queue du groupe cercariaea (C). Ce dernier type de cercaire est le plus fréquemment rencontré : $46 \%$ des Mollusques parasités l'hébergent, il appartient à une espèce de Trématode qui présente un large spectre d'hôtes intermédiaires $(5$ contre 1 ou 2 pour les autres espèces) comprenant à la fois des Prosobranches et des Pulmonés : V. cristata, V. pulchella, V. piscinalis, $G$. albus, $P$. planorbis. D'autre part, deux infestations mixtes ont été observées chez $L$. peregra : l'une avec des xiphidiocercaires X2 et X3, l'autre avec des furcocercaires $\mathrm{F} 2$ et F3.
Parmi les huit espèces de Gastéropodes hôtes, P. planorbis associe une prévalence globale de Trématodes relativement élevée $(32 \%)$ et une abondance qui la place au troisième rang dans la communauté de Gastéropodes (4,16\% des Mollusques parasités sont des $P$. planorbis). De plus, ce Mollusque héberge trois espèces différentes de Trématodes, parmi lesquelles les types " xiphidiocercaire $\mathrm{X} 1$ " et " cercariaea $\mathrm{C}$ " qui représentent à eux seuls $78 \%$ de la communauté parasitaire.

\section{DISCUSSION}

$\mathrm{P}$ armi les 35 espèces de Gastéropodes dulcicoles enregistrées en France, dont 25 en Illeet-Vilaine (Baudet et al., 1988; Costil, 1993), 15 ont été trouvées dans l'étang de Combourg (dont 12 déjà signalées en Ille-et-Vilaine). Les espèces manquantes sont pour la plupart des espèces rares (Anisus rotondatus, Anisus vortex, Physa fontinalis, Aplexa hypnorum, Bithynia laechii, Viviparus viviparus) ou surtout présentes dans le Sud du département (Bathyomphalus contortus, Menetus dilatatus) et qui n'affectionnent pas les eaux stagnantes (vs courantes). Les 15 espèces enregistrées se répartissent dans quatre familles de Pulmonés (Planorbidae, Lymnaeidae, Physidae, Ancylidae) et une seule famille de Prosobranches (Valvatidae), la famille des Planorbidae étant la mieux représentée avec six espèces et près de $76 \%$ des individus récoltés. G. albus, planorbe la plus fréquente en Ille-et-Vilaine (Costil, 1993), représente 


\begin{tabular}{|c|c|c|c|c|}
\hline Parasites & & Fréquence \% & Spectre d'hôtes & Effectif hôtes \\
\hline \multirow[t]{3}{*}{3 xiphidiocercaires } & $\mathrm{X} 1$ & 32,20 & $\mathrm{Pp} ; \mathrm{Pc}$ & 19 \\
\hline & $\mathrm{X} 2$ & 3,39 & Lp & 2 \\
\hline & $\mathrm{X} 3$ & 3,39 & Lp & 2 \\
\hline \multirow[t]{3}{*}{3 furcocercaires } & $\mathrm{F} 1$ & 1,69 & Ga & 1 \\
\hline & $\mathrm{F} 2$ & 3,39 & Lp & 2 \\
\hline & F3 & 8,47 & Lp; Ls & 5 \\
\hline 1 échinostome & $\mathrm{E}$ & 8,47 & Pp; Ls & 5 \\
\hline 1 notocotyle & $\mathrm{N}$ & 1,69 & $\mathrm{Ga}$ & 1 \\
\hline 1 cercariae & $\mathrm{C}$ & 45,76 & $\mathrm{Vc} ; \mathrm{Vpu} ; \mathrm{Vpi} ; \mathrm{Ga} ; \mathrm{Pp}$ & 27 \\
\hline
\end{tabular}

(Ga : Gyraulus albus, Lp : Lymnaea peregra, Ls : Lymnaea stagnalis, Pc : Planorbarius corneus, Pp : Planorbis planorbis, Vc : Valvata cristata, Vpi : Valvata piscinalis, Vpu : Valvata pulchella).

Tableau IV. - Communauté de Trématodes de l'étang de Combourg : fréquence des parasites, spectre d'hôtes et effectif des Mollusques parasités (59 au total).

$67 \%$ des Gastéropodes prélevés dans l'étang de Combourg. Les fluctuations saisonnières de la dynamique des populations de Mollusques expliquent la faible abondance de certaines espèces telles que les limnées et les planorbes cornées pendant la période froide de l'année, alors que la rareté d'autres espèces comme $P$. acuta, A. lacustris, G. laevis ou S. nitida est caractéristique de ce peuplement malacologique quelle que soit la période de l'année. D'autre part, la richesse spécifique de cet étang (15) est nettement supérieure à la valeur moyenne des étangs d'Ille-et-Vilaine $(5,7 \pm 0,7)$ (Costil, 1993). L'étang de Combourg constitue donc un site d'étude très intéressant, non seulement en raison de la richesse et de la diversité de la communauté de Gastéropodes, mais aussi de celles de la communauté de Trématodes, comme en témoignent les neuf types de cercaires répertoriés.

Le taux de parasitisme que nous avons enregistré varie énormément selon que l'on considère l'ensemble du peuplement malacologique : 4,32\% (59 individus parasités sur 1365 individus examinés quelle que soit l'espèce), ou chacune des populations en présence : jusqu'à $80 \%$ de Mollusques parasités pour L. stagnalis. Selon Anderson \& May (1979), la prévalence des Trématodes larvaires dans les populations naturelles de Gastéropodes n'excèderait pas 4 à $5 \%$, cependant, beaucoup de travaux ont montré qu'elle peut être très élevée, jusqu'à $95 \%$ chez certaines populations de Mollusques (Lafferty, 1993 b, Lauckner,1986; Crews \& Esch, 1986). La grande variabilité des valeurs recueillies est liée à l'écologie de la transmission (fluctuations saisonnières de la prévalence en fonction des périodes de recrutement du parasite). Ce sont les fluctuations de la prévalence qui permettent de quantifier les changements de taille de la population parasite. Selon Erasmus (1972) et Crews \& Esch (1986), il existe généralement deux pics de prévalence chez les Mollusques aquatiques d'Europe : à la fin du printemps (mai-juin) et de l'été (septembre-octobre), qui sont principalement dus aux changements qui surviennent dans la faune malacologique et qui restent à vérifier pour la communauté de Mollusques de l'étang de Combourg. Beaucoup d'autres facteurs abiotiques et biotiques peuvent intervenir dans les fluctuations de la prévalence, parmi lesquels les caractéristiques du milieu (température, végétation, eau stagnante/courante...), le comportement des hôtes intermédiaires et définitifs, leur cycle de développement... Plusieurs auteurs ont par exemple mis en évidence en milieu naturel (Dubois, 1929; Sousa, 1983; Minchella et al., 1985; Goater et al., 1989; Manga-Gonzalez et al., 1994) et au laboratoire (Anderson \& Crombie, 1984) une relation entre la prévalence et la taille des Mollusques, qui atteste l'importance de la structure d'âge de la population hôte. Même si certaines espèces de Trématodes peuvent induire chez leur Mollusque hôte un phénomène qualifié de gigantisme par MacClelland \& Bourns (1969), Wilson \& Denison (1980) et Joosse \& Van Elk (1986), il semble que le plus souvent ce sont les Mollusques âgés vs jeunes qui sont les plus parasités en raison d'un temps d'exposition au parasite supérieur. Toutefois, nous n'avons pas mis en évidence de différences significatives de taille entre Mollusques sains et parasités de même espèce.

En ce qui concerne le potentiel régulateur des Trématodes vis-à-vis des populations de Mollusques, la relation inverse entre la fréquence des Gastéropodes et le taux de prévalence des Trématodes, jusqu'alors non démontrée en pratique à l'échelle communautaire, permet d'envisager un impact non négligeable du parasitisme sur la dynamique des populations hôtes. Parmi les espèces qui se distinguent par leur forte prévalence (tableau II), P. planorbis, dont l'effectif n'apparaît pas limitant, constitue un modèle d'étude particulièrement intéressant pour tester l'hypothèse d'une régulation des populations par les Trématodes. S'agis- 
sant d'un Mollusque de grande taille, il est de surcroît plus facile d'accès sur le terrain et permet le marquage dans le cadre de capture-recaptures multiples. Il faut cependant rester prudent quant au choix du (ou des) modèle(s) d'étude, dans la mesure où d'une année sur l'autre, la composition faunistique peut présenter des changements très importants, en particulier chez les Mollusques, même s'ils constituent toujours environ $80 \%$ de la biomasse des Invertébrés dans un écosystème dulcicole (Mouthon, 1980).

\section{RÉFÉRENCES}

ANDERSON R.M. Regulation of host population growth by parasite species. Parasitology, 1978, 76, 119-157.

ANDERSON R.M. Parasite pathogenecity and the depression of host population equilibria. Nature, 1979, 279, 150-152.

ANDERSON R.M. \& MAY R. Regulation and stability of host-parasite population interactions. I. Regulatory processes. Journal of animal ecology, 1978, 47, 219-249.

Anderson R.M. \& MAY R. Prevalence of schistosome infections within molluscan populations: observed patterns and theoretical predictions. Parasitology, 1979, 79, 63-94.

Anderson R.M. \& Crombie J. Experimental studies of age-prevalence curves for Schistosoma mansoni infections in populations of Biomphalaria glabrata. Parasitology, 1984, 89, 79-104.

Baudet J., Gruet Y. \& maillard Y. Distribution de certaines espèces de la malacofaune du marais breton-vendéen (Loire-Atlantique et Vendée). Haliotis, 1988, 18, 21-31.

BAyne C.J. \& Loker E.S. Survival within the snail host, in : The Biology of Schistosomes: From Genes to Latrines. Rollinson D. \& Simpson A.J.G. (eds), Academic Press, London, 1987, 321-342.

Combes C. Parasitisme et équilibre des écosystèmes. REED (Ministère de l'Environnement), 1993, n 43-44 (juilletdécembre), 26-28.

Costıl K. Contribution à l'écologie des Mollusques Gastéropodes dulcicoles armoricains, recherches bio-écologiques et écophysiologiques sur deux espèces de Pulmonés Planorbidae: Planorbarius corneus (L.) et Planorbis Planorbis (L.). Thèse de l'Université de Rennes I, 1993, 421 p.

Crews A.E. \& Esch G.W. Seasonal dynamics of Halipegus occidualis (Trematoda: Hemiuridae) in Helisoma anceps and its impact on fecundity of the snail host. Journal of Parasitology, 1986, 72 (5), 646-651.

Dobson A.P. \& Hudson P.J. Parasite, disease and the structure of ecological communities. Trends in ecology and evolution, 1986, 1, 11-15.

DoBson A.P. \& Hudson P.J. Regulation and stability of a freeliving host-parasite system: Trichostrongylus tenuis in red grouse. II. Population models. Journal of animal ecology, 1992, 61, 487-498.

Dubois G. Les cercaires de la région de Neuchâtel. Bulletin de la Société Neuchâteloise de Sciences Naturelles, 1929, 53, 3-177.
ERASmus D.A. The digenetic cycle-larval forms, in : The biology of Trematodes. Edward Arnold Publishers (eds), 1972, 21-27.

Goater T.M., Shostak A.W., Williams J.A. \& Esch G.W. A mark-recapture study of trematode parasitism in overwintered Helisoma anceps (Pulmonata), with special reference to Halipegus occidualis (Hemiuridae). Journal of Parasitology, 1989, 75 (4), 553-560.

Holmes J.C. Impact of infectious disease agents on the population growth and geographical distribution of animals, in : Population biology of infectious diseases. Anderson R.M. \& May R.M. (eds) Springer-Verlag, 1982, 37-51.

Hudson P.J., Dobson A.P. \& Newborn D. Cyclic and noncyclic populations of red grouse: a role for parasitism? in : Ecology and genetics of host-parasite interactions. Rollinson D. \& Anderson R.M. (eds), Academic press, London, $1985,77-89$.

Hudson P.J. \& DoBson A.P. Regulation and stability of a freeliving host-parasite system: Trichostrongylus tenuis in red grouse. I. Monitoring and parasite reduction experiments. Journal of animal ecology, 1992, 61, 477-486.

Hurd H. Physiological and behavioural interactions between parasites and invertebrate hosts. Advances in parasitology, 1990, 29, 271-318.

Joosse J. \& VAn ElK R. Trichobilharzia ocellata: Physiological characterization of giant growth, glycogen depletion, and absence of reproductive activity in the intermediate snail host, Lymnaea stagnalis. Experimental Parasitolology, 1986, 62, 1-13.

Keymer A.E., Gregory R.D., Harvey P.H., Read A.F. \& SkorPING A. Parasite-Host ecology: case studies in population dynamics, life-history evolution and community structure. Acta Oecologica,1991, 12 (1), 105-118.

LAFFERTY K.D. Effects of parasitic castration on growth, reproduction and population dynamics of the marine snail Ceritbidea californica. Marine ecology progress series, $1993 a, 96,229-237$.

LAFFERTY K.D. The marine snail, Cerithidea californica, matures at smaller sizes where parasitism is high. Oikos, 1993 b, 68, 3-11.

LAUCKNER G. Ecological effects of larval trematode infestation on littoral marine invertebrate populations. International journal for parasitology, 1986, 17, 391-398.

McClelland G. \& Bourns T.K.R. Effects of Trichobilharzia ocellata on growth, reproduction, and survival of Lymnaea stagnalis. Experimental Parasitology, 1969, 24, 137-146.

Manga-Gonzalez Y., Gonzales-Lanza C. \& Kanev I. Lymnaea truncatula, intermediate host of some Plagiorchiidae and Notocotylidae species in Leon, NW Spain. Journal of Helminthology, 1994, 68, 135-141.

MAY R.M. Parasite infections as regulators of animal populations. American Scientist, 1983, 71, 36-45.

Minchella D.J., Leathers B.K. \& Brown K.M. Host and parasite counteradaptation: an example from a freshwater snail. American Naturalist, 1985, 126, 843-854.

Mouthon J. Contribution à l'écologie des Mollusques des eaux courantes. Esquisse biotypologique et données écologiques. Thèse de l'Université de Paris VI, 1980, 169 p. 
SCOTT M.E. \& ANDERSON R.M. The population dynamics of Gyrodactylus bullaturudis on guppies. Parasitology, 1984, 89, 159-194.

Scotr M.E. Regulation of mouse colony abundance by Heligmosomoides polygyrus. Parasitology, 1987, 95, 111-124.

SousA W.P. Host life history and the effect of parasitic castration on growth: a field study of Ceritbidea californica Haldeman (Gastropoda: Prosobranchia) and its Trematode parasites. Journal of experimental marine biology and ecology, 1983, 73, 273-296.

Thompson N.S. Metabolic integration during the host associations of multicellular animal endoparasites. Comparative biochemistry and physiology, 1985, 81B (1), 21-42.

WILSON R.A. \& Denison J. The parasitic castration and gigantism of Lymnaea truncatula infected with the larval stages of Fasciola bepatica. Zeitschrift für Parasitenkunde, 1980, 61, 109-119.

Reçu le 21 septembre 1996 Accepté le 20 décembre 1996 